\title{
Pratiques
}

Linguistique, littérature, didactique

\section{Les livres de poèmes illustrés : production littéraire et lecture de quelques maitres en formation}

Books of illustrated poems: literary production and reading of some masters in formation

\section{Christine Boutevin}

\section{(2) OpenEdition}

Journals

Édition électronique

URL : http://journals.openedition.org/pratiques/3596

DOI : $10.4000 /$ pratiques.3596

ISSN : 2425-2042

Éditeur

Centre de recherche sur les médiations (CREM)

\section{Référence électronique}

Christine Boutevin, «Les livres de poèmes illustrés : production littéraire et lecture de quelques maitres en formation », Pratiques [En ligne], 175-176 | 2017, mis en ligne le 22 décembre 2017, consulté le 19 avril 2019. URL : http://journals.openedition.org/pratiques/3596 ; DOI : 10.4000/ pratiques.3596

Ce document a été généré automatiquement le 19 avril 2019

(c) Tous droits réservés 


\title{
Les livres de poèmes illustrés : production littéraire et lecture de quelques maitres en formation
}

\author{
Books of illustrated poems: literary production and reading of some masters in \\ formation
}

Christine Boutevin

1 Depuis une vingtaine d'années, le corpus scolaire d'œuvres littéraires est préconisé au moyen de répertoires qui visent à aider les enseignants dans leurs choix de supports de lecture en classe. La poésie ne fait pas exception. En effet, à la suite des instructions officielles de 1995, la bibliographie de La Bibliothèque des mille et un livres ${ }^{1}$ proposait une trentaine d'anthologies et de recueils pour guider les maitres. Actuellement, le corpus s'est élargi puisque, dans les listes de référence de 2012, les instances officielles recommandent 58 titres dans la rubrique « poésie » (21 pour le cycle 2 et 37 pour le cycle $\left.3^{2}\right)$.

2 Or cet ensemble que nous appellerons « livres de poème(s) illustrés » est caractérisé par son hybridité (Connan-Pintado \& Poulou, 2008, p. 8-10). Dans pratiquement toutes les publications se trouvent un ou plusieurs poèmes accompagnés d'images. Ce type de livre n'est pas inconnu de la recherche en littérature. Il a été étudié sous des appellations diverses: grand livre illustré (Chapon, 1987), livre de dialogue (Peyré, 2001), livre d'artistes (Moeglin-Delcroix, 2006). Cependant en littérature de jeunesse, les spécialistes bien qu'ils aient décrit la complexité des rapports entre texte et image dans les albums, ont laissé de côté les livres de poème(s) illustrés.

3 C'est pourquoi nous proposons une analyse de ces publications au double langage sémiotique, à la fois verbal et plastique, en prenant en compte les apports de la sémiologie, de l'histoire des arts du livre, de la poétique et de l'album pour la jeunesse. Notre hypothèse est que l'existence de tels ouvrages dans les listes officielles révèle les tensions à l'œuvre aujourd'hui dans l'enseignement de la poésie à l'école, premièrement 
tension entre œuvre du patrimoine et œuvre contemporaine, deuxièmement tension entre lecture/littérature et histoire des arts.

Par ailleurs, la présence d'un tel corpus dans l'enseignement élémentaire est une situation nouvelle: comment les maitres qui constituent les premiers lecteurs de ces ouvrages lisent-ils ce type d'œuvres mêlant poésie et art visuel? On sait quelles représentations ils se font de l'album (Leclaire-Halté et al., 2009) et les difficultés qu'ils ont à faire lire un ensemble réunissant deux systèmes sémiotiques. De plus, le genre poétique présente des difficultés de lecture qui lui sont propres, liées à des zones d'ombre, d'incompréhension, de non-sens (Doumet, 2004 ; Favriaud et al., 2011 ; Siméon, 2012), difficultés qui mettent en cause l'image de soi. Ces constats nous interrogent sur la lecture littéraire à mettre en œuvre pour ces ouvrages hybrides. Aussi, nous montrerons comment la lecture subjective reposant sur les théories du sujet lecteur (Langlade \& Fourtanier, 2007 ; Rannou, 2010 ; Rouxel \& Langlade, 2004) permet, dans une certaine mesure, de comprendre comment des lecteurs en formation abordent ces œuvres dans leur double dimension textuelle et visuelle.

Dans cet article, nous présentons donc une typologie des livres de poème(s) illustrés. Nous distinguons les quatre catégories littéraires suivantes: l'anthologie illustrée et le poème-album dans lesquels l'illustration permet de lire autrement les productions du passé ; le recueil de création illustré et l'album-poème, lieux privilégiés de la rencontre entre poètes et artistes contemporains. Après l'analyse de ces différents supports, nous évoquons un dispositif expérimenté en formation dans le cadre du master MEEF. À titre exploratoire, nous verrons comment les étudiants traitent les images d'un album-poème, intitulé Les Étoiles sont tombées (David \& Propeck, 2007), de quelle manière ils se représentent un élément textuel énigmatique en s'intéressant plus ou moins à l'iconographie de l'œuvre et quelles perceptions de la guerre, sujet de l'œuvre, ils ont pu construire par la lecture de cette œuvre double.

\section{Typologie des livres de poème(s) illustrés}

\section{L'anthologie illustrée}

Dans les listes officielles de 2012, l'anthologie se trouve représentée par 17 titres. Elle regroupe des textes soit d'un seul, soit de plusieurs auteurs. Cette forme éditoriale a longtemps dominé dans la production de la poésie pour la jeunesse (Ballanger \& Heise, 1995) notamment parce que l'école en est une grande consommatrice. On note cependant un changement d'orientation dans les choix de l'institution. Alors qu'était privilégiée l'anthologie thématique en petit format sans illustration, aujourd'hui on lui préfère l'album consacré à un poète en particulier ou regroupant une grande variété de poèmes choisis selon des critères variés (forme poétique, origine géographique, chronologie...).

7 Or, la collection « La poésie » de Rue du monde est particulièrement représentative de ces anthologies sous forme d'album, dont les trois titres suivants: Il pleut des poèmes (Henry, 2003), Tour de terre en poésie (Henry, 1998) et Le Tireur de langue (Henry, 2000) appartiennent au corpus de l'école élémentaire depuis 2002. Les critères de l'anthologie (Fraisse, 1997, p. 94) définis par J.-M. Henry à qui l'éditeur, A. Serres, a confié la collection, traduisent le refus manifeste de glorifier certains auteurs. Aux poètes du panthéon, sont préférées les figures des poètes du monde entier, qui écrivent dans toutes les langues même les plus minoritaires. De plus la collection se présente sous un format à 
l'italienne $(19 \times 25 \mathrm{~cm})$ qui n'est pas anodin puisqu'il traduit l'idée d'ouverture véhiculée par cette maison d'édition. Lorsqu'on le déploie, le livre ressemble à un carnet de voyage, ce qu'il est symboliquement: voyage à travers les mots, les langues, les pays, les générations, les formes poétiques. Il invite ainsi à s'interroger sur la poésie elle-même et sur la poésie pour l'enfance et la jeunesse en particulier.

Enfin, la plupart du temps, l'illustration structure l'espace de la double page. Par le cadre, le fond, les motifs, elle constitue un environnement pour les poèmes. Le volume intitulé Il pleut des poèmes par exemple présente des textes disposés sur des lignes obliques, dans un mouvement ascendant de la gauche vers la droite et la typographie alterne le bleu et le noir. Le nombre de poèmes est croissant jusqu'à la double page centrale puis décroissant. En bas de page, Zaü dessine la terre (campagne, mer, ville) qui reçoit ce don du ciel, des poèmes très brefs de toutes les couleurs du monde. L'anthologie devient ainsi un écrin dont la part illustrée indique explicitement le destinataire. En effet, de manière plus globale, les motifs représentés dans ces ouvrages (des enfants, des animaux, des maisons...), les techniques employées (dessins imitant des formes enfantines) sont autant d'indices utilisés pour atteindre le lectorat visé. L'illustration fait partie de la stratégie de communication de la collection.

Ces anthologies se révèlent donc en accord avec les textes officiels qui, pour l'approche de la littérature de jeunesse, recommandent d'être attentif à la production éditoriale contemporaine présentant des illustrations dont il faut savoir comprendre les fonctions. En outre, le dossier en ligne "la poésie à l'école ${ }^{3}$ " affirme qu'il est nécessaire de confronter les élèves à la diversité des genres, des lieux de production, des époques. Enfin la variété des questionnements proposés par ces anthologies autour des mots, des origines, des nations, invite à la réflexion. Or, il ne s'agit plus aujourd'hui d'envisager la poésie seulement comme support pour la récitation, ni uniquement comme inducteur de jeux d'écriture. Les poèmes, comme le souligne le document ressource pour la culture littéraire, "supposent le même travail de compréhension et conduisent aux mêmes débats interprétatifs ${ }^{4} »$ que les autres genres littéraires.

\section{Le poème-album}

10 Le mot album, dans sa relation avec la poésie, présente de nombreux aspects. Dans l'histoire de la littérature, durant la Renaissance, il est question d' "album amicorum ", recueil manuscrit de pièces de vers issues des rencontres mondaines qui se tenaient autour d'une figure féminine, protectrice des poètes. Plus tard, on parlera d'album d'artistes réunissant un poète et un peintre (par exemple F. Ponge [1945] et J. Dubuffet, Saint-John Perse [1962] et G. Braque). Aujourd'hui l'album pour enfant reconfigure la notion.

11 En effet, dans le champ de la littérature de jeunesse, I. Nières-Chevrel (2012) précise que, pour l'édition, l'album est devenu un support dans lequel la part visuelle est essentielle. Elle préfère conserver le terme d'illustration dans le cas où il s'agit d'« une création graphique qui vient s'ajouter » (ibid., p. 19) à un texte antérieur et autonome. Le poèmealbum correspond à cette conception, puisqu'à l'origine, le texte poétique existe en dehors de tout accompagnement iconographique. Il est extrait par l'éditeur d'un recueil autographe déjà publié, appartenant à la littérature générale. Imprimé sur les doubles pages de l'album et non plus sur la page d'un recueil, le poème se trouve isolé de son ensemble, dans une nouvelle configuration visuelle. Il perd ainsi sa disposition tabulaire 
initiale. On peut alors parler d'une forme d'adaptation (Louichon, 2008) au moyen de cette mise en page. Dans un deuxième temps, l'artiste illustrateur propose une lecture du poème dans son langage plastique. Cette seconde modalité d'adaptation entre en relation avec le support, et le texte original devient alors poème-album.

Dans l'histoire du livre de poésie pour enfants, ce type est plus récent que celui de l'anthologie illustrée. Cependant, l'un et l'autre présentent deux points communs: l'existence de poèmes autonomes et la présence d'une illustration seconde. Les listes officielles en vigueur actuellement proposent cinq titres que l'on peut trouver sous la forme de sept poèmes-albums : pour le cycle 2, Zoo de M. Butor, On peut se tromper de Norge et La Pêche à la baleine de J. Prévert (1979); pour le cycle 3, Liberté de Paul Éluard (1997 ; 2012) et L'Ogre de Moscovie de V. Hugo (2008a et b).

Quelles sont les finalités de ce nouvel objet culturel ? Disons tout d'abord que les poèmesalbums se trouvent dans le corpus de littérature pour les enfants de 6 à 11 ans parce qu'ils constituent des adaptations de textes publiés par des auteurs classiques et/ou reconnus par l'institution littéraire. Ce type de livre donne accès aux plus jeunes à la poésie légitimée par des instances extérieures à l'école, notamment par les grands éditeurs et par l'Académie française, et contribue à la transmission du patrimoine littéraire, ainsi que des auteurs contemporains que les élèves de l'école élémentaire pourront retrouver au niveau supérieur. De plus, dans ces livres, l'intérêt de l'image n'est pas le même selon que l'on se place du côté de l'illustrateur ou du jeune lecteur. Tandis que la partie imagée est seconde dans le temps de la création, au moment de la lecture elle surgit d'emblée pour le lecteur (qui selon l'âge ne l'est pas encore parfois), car elle lui impose son monde. L'illustration constitue donc un enjeu important pour l'entrée en poésie à l'école, étant donné son pouvoir de fascination. Spécifiquement publié pour les enfants, le poèmealbum est un outil de diffusion des textes poétiques, au sein duquel l'illustration joue un rôle de « régulation " (Ceysson, 1997, p. 20-29) : elle est un signe de communication de la poésie pour l'enfance et la jeunesse. Mais il ne faudrait pas la réduire à cette finalité, car le poème-album permet aux élèves de lire la poésie d'hier devenue source d'inspiration pour les artistes d'aujourd'hui. Il peut donc enfin être un support d'apprentissage intéressant pour l'enseignement de l'histoire des arts, non seulement parce qu'il réunit art du langage et art plastique, mais aussi parce qu'il montre quelle relation l'un et l'autre nouent par-delà les générations.

\section{Le recueil illustré}

Dans son acception générale, le terme "recueil» désigne un livre de poésie où sont rassemblés des textes, le but étant de constituer un ouvrage à part entière. Cependant à l'instar de D. Combe (2002), il convient de distinguer l'anthologie qui réunit des poèmes, à l'origine dispersés dans plusieurs livres, du recueil individuel autographe. Ce dernier est une œuvre de création pensée par un poète comme un tout. En outre, on note que tous les recueils poétiques recommandés par les instances officielles sont illustrés. L'artiste plasticien est donc partie prenante dans la conception de ces œuvres. Contrairement aux deux catégories définies précédemment, le recueil illustré appartient à la création contemporaine (Boutevin, 2015). Les productions proposées à la lecture scolaire datent pour les plus anciennes des années 1990.

Venons-en désormais à envisager le recueil en tant que création verbale, poétique et iconique, artistique. En effet, l'auteur n'est pas le seul maitre de la composition finale. 
Comme I. Langlet (2003), nous nous interrogeons sur les acteurs du recueil. Si le poète écrit et organise les textes, l'illustrateur, mais aussi l'éditeur participent pleinement à la conception. F. Chapon (1987) et Y. Peyré (2001) évoquent longuement cette triple entente entre poète, peintre et éditeur dans le cas du grand livre illustré ou du livre de dialogue. Qu'en est-il pour le livre de poèmes illustré destiné aux enfants? Quel est le rôle de chacun?

Sans dresser une liste exhaustive des "facteurs configuratifs»(Combe, 2002, p. 18), disons tout d'abord que du côté de l'auteur, la/les forme(s) poétique(s), les titres (titres $\mathrm{du}$ recueil, des poèmes, des sections), la thématique et la logique d'ordonnancement sont autant d'éléments qui créent l'unité du livre. Le poète, par ces différents gestes créateurs, commence à donner forme à l'œuvre: il montre par là qu'il envisage la lecture de ses poèmes dans un tout, parfois complexe d'ailleurs, et dans un espace dont il n'est pas le seul responsable.

17 Le rôle des éditeurs est également important et plusieurs d'entre eux se consacrent entièrement à la poésie (MØtus, Soc \& Foc, Cheyne). Leur intervention lors de la composition du livre fait que le lecteur a entre les mains un ensemble cohérent, une unité visible notamment grâce aux trois éléments suivants : la collection, la typographie et la mise en page. Certes le lecteur est libre de construire son cheminement dans le recueil, cependant les caractéristiques éditoriales induisent certains gestes et orientent la lecture. Les éditeurs sont d'ailleurs bien souvent poètes eux-mêmes, L. Dubost (Le dé bleu), J. Brière (La Renarde rouge), ce qui explique, pour une part, que leur intervention participe pleinement de l'œuvre.

Enfin comment l'illustration peut-elle contribuer à donner aux lecteurs une impression d'ensemble? Dans ses analyses de l'album contemporain, S. Van der Linden $(2006 ; 2013)$ mentionne la variété et la richesse des techniques présentes dans la production actuelle pour la jeunesse. Le recueil illustré en témoigne également. Mais la variété des techniques utilisées ne contredit pas l'effet de cohésion, car à une œuvre correspond toujours la même technique. De plus, même si la plupart du temps, des poèmes différents occupent chaque page ou chaque double page, les illustrations présentent des motifs récurrents (un petit chien noir anthropomorphisé dans Super Gloupi [Vendel, 2014] ; des empreintes végétales vert clair dans Le Bestiaire des mots [Serres, 1988]). Enfin certains emplacements sont choisis pour marquer un seuil et les illustrations peuvent servir d'ornement et structurer le recueil en participant ou non à sa signification.

19 Cette brève analyse du recueil illustré montre combien il est nécessaire de construire auprès des élèves des compétences générique, paratextuelle, interprétative pour la lecture des poèmes, du support et des illustrations. Le caractère hybride de ces œuvres qui constituent la majorité du corpus scolaire, entraine des lectures qui, dans l'enseignement de la poésie, devraient prendre en charge les aspects lisibles et visibles, tout comme notre dernière catégorie, l'album-poème.

\section{L'album-poème}

R. Lefort est l'un des premiers chercheurs en littérature à s'être intéressé à l'image dans le livre de poésie pour l'enfance et la jeunesse. Nous reprenons le concept d'« albumpoème » qu'il a défini ainsi :

Dans l'album-poème, il ne s'agit plus de lien de dépendance de l'un vis-à-vis de l'autre, du texte vis-à-vis de l'image ou inversement, mais de faire en sorte que le 
lieu du poème soit pleinement le livre, que ce soit cet « espace de résonance » dont nous avons parlé. (Lefort, 2008, p. 30) catégorisation problématique, d'ailleurs on ne le trouve pas nécessairement dans la rubrique "poésie ». Prenons l'exemple de l'œuvre de F. David et A. Gauthier, Est-elle Estelle? (2002), présente parmi les albums, depuis 2007, dans la liste pour des élèves de CPCE1. Les aspects plastiques et visuels, essentiels dans l'ouvrage, font que la vue est sollicitée en priorité, tant pour l'observation des images, que pour la lecture du poème qui se dessine page après page. La logique organisationnelle de l'ensemble repose sur un jeu d'échos entre texte et image. Dès la couverture, on s'interroge sur la représentation d'une figure féminine dont la chevelure forme un point d'interrogation. À chaque double page, le lecteur se questionne verbalement et plastiquement à propos de l'identité de cette figure. Or, il ne peut donner du/des sens à l'œuvre qu'à la dernière double page où apparait le poème dans son intégralité : une sorte de panneau d'ophtalmologue à l'envers, transformé en poème-affiche, vers lequel les regards dessinés par A. Gauthier se tournent. L'album-poème devenu jeu entre le lecteur, le poète et l'artiste réinterroge le genre luimême de la poésie pour l'enfance et la jeunesse.

De fait, la présence de cette catégorie (nouvelle) parmi les préconisations scolaires montre que les instances officielles ont fait des choix qui questionnement les normes littéraires que l'école impose elle-même en matière de poésie. Une autre œuvre, Rêve-moi une lettre d'A. Bertier (2005), se révèle, à l'analyse, être une véritable expérience poétique, et non un simple abécédaire. Les images « écrites » et les lettres « imagées » de l'artiste, bien au-delà de l'apprentissage de l'alphabet, introduisent l'enfant dans un univers imaginaire et l'initient à un art du langage qui unit lettres et formes, écriture et objet, mot et musique. On pourrait citer aussi C'est corbeau de J.-P. Dubost et K. Couprie (1998), carnet poétique où s'entrelacent les noirs de l'artiste et les notes rétrospectives du poète, évoquant l'un et l'autre la vie d'un freux égaré parmi les humains. Cette œuvre en prose à mi-chemin entre le recueil illustré et le recueil-album ébranle non seulement les codes de la poésie pour la jeunesse, mais également l'esthétique de l'image dans l'album pour 
enfant, par l'usage exclusif du noir et blanc et de motifs grossièrement représentés. L'album-poème parait donc bien adapté pour une approche du livre en tant qu'œuvre d'art double, poétique et visuel, pour l'enseignement de l'histoire des arts, avec comme réserve une méconnaissance de ce type d'œuvre de la part des enseignants. Ce constat nous a donc conduite à mener l'expérience présentée ci-dessous.

\section{Lecture d'un album-poème en formation}

La plupart des futurs enseignants du premier degré ont pratiqué la lecture de la poésie uniquement dans le contexte de l'enseignement primaire et secondaire, avec des approches centrées sur les genres, les formes et l'histoire littéraire. Or, la lecture littéraire dans les programmes, depuis 2002, ne préconise pas seulement une lecture distanciée, mais réclame que celle-ci soit articulée à une lecture participative ${ }^{5}$ mobilisant les sens, les émotions, l'imagination.

Par conséquent, nous avons souhaité expérimenter une lecture subjective de la poésie en situation empirique, dans le cadre de la formation des enseignants du premier degré, car la lecture professionnelle dépend de la lecture personnelle (Butlen et al., 2008). Le présupposé de la lecture subjective est que le lecteur qui s'approprie le poème peut élaborer du sens, des sens à travers ses configurations imageantes, fantasmatiques, axiologiques (Lacelle \& Langlade, 2007). Dans cette lecture, on considère que connaissance et expérience sont étroitement liées et que le savoir se construit sur le vécu affectif, sensoriel, effectif du lecteur.

De plus, notre hypothèse principale est que l'objet littéraire lui-même ne suffit pas : la lecture subjective du livre de poème(s) illustré s'apprend et certains dispositifs didactiques nous semblent plus adéquats que d'autres, notamment ceux qui privilégient l'expression personnelle partagée, car ils peuvent amener les lecteurs à « comprendre » le poème et son image, c'est-à-dire à les embrasser, à les habiter.

Notre terrain d'expérience est celui du master 2, en 2012, à l'Université Bordeaux 4 IUFM d'Aquitaine. Nous nous sommes adressée à deux enseignants, formateurs de français, spécialistes de leur discipline, qui ne font pas de recherche, et leur avons proposé de lire l'album-poème, Les Étoiles sont tombées (David \& Propeck, 2007), sans indication préalable (voir texte en annexe). L'œuvre a été mise à leur disposition durant deux semaines. Puis nous avons mené un entretien préparatoire afin de présenter et d'expliquer aux deux formateurs le dispositif de la séance de lecture que nous avions construit et expérimenté nous-même antérieurement, dispositif reposant sur les notions de sujet lecteur et de lecture subjective, théorisées par A. Rouxel (2004), G. Langlade et M.-J. Fourtanier (2007). En procédant ainsi, notre position est celle d'une observatrice extérieure à la mise en œuvre.

Deux groupes sont concernés, dans deux contextes d'enseignement légèrement différents : le premier constitué de onze étudiantes dans les Landes, en deuxième année de master. Pour ce groupe, la lecture des Étoiles sont tombées a eu lieu dans le cadre d'une unité d'enseignement consacrée à la didactique du français. Le second, dans les PyrénéesAtlantiques, suivait un cours optionnel sur la littérature de jeunesse, en première année. Quatorze étudiantes y participaient. Au total, notre observation porte sur vingt-cinq lectrices pour qui il s'agissait d'expérimenter la lecture d'un album-poème, un type de 
livre problématique du point de vue générique et complexe du point de vue de l'interdépendance du texte et de l'image.

31 L'album, en noir et blanc, présente un texte qui se déploie sur vingt-et-une pages, en regard duquel se trouvent vingt-et-une photographies d'A. Propeck qui n'ont pas été réalisées pour l'album, ce que les étudiantes ignorent. Le poète a sélectionné certains clichés, complètement autonomes avant la réalisation de l'album-poème. Pourtant, la réciprocité visuelle apparait dans la réalisation de cette œuvre double qui propose une succession de doubles pages sur lesquelles l'image à gauche et le texte à droite créent un espace dialogique. Le poème parle des effets de la guerre :

[...] Quand il y a la guerre, elle m'a dit,

plus rien ne tient en équilibre.

Il y a des montagnes de barbelés pour faire tout tenir de force quand il y a la guerre.

Quand il y a la guerre, elle m'a dit, il y a des pièges partout.

Et les ballons légers des jeux sont attrapés comme des rats. [...]

Mais les photographies ne montrent jamais les hommes, ni soldat ni civil, ni blessé ni mort. Les mots et les images ne représentent pas cette guerre, mais leur association, dans la suggestion, dit l'horreur.

Trois éléments nous ont paru essentiels pour justifier la présence de cet objet sémiotique particulier dans un dispositif de lecture subjective : l'étrangeté du livre lui-même et de son genre littéraire, le caractère " résistant » (Tauveron, 1999) du texte et des images et la capacité de cet album-poème à susciter des émotions fortes.

Le dispositif général de la séance dont nous détaillons la cinquième étape est le suivant :

- $1^{\text {re }}$ étape : individuellement lecture silencieuse de l'œuvre

- $2^{\mathrm{e}}$ étape : individuellement expression de ses premières impressions à l'écrit

- $3^{\text {e }}$ étape : relecture, choix d'une double page et lecture à haute voix du texte retenu par chacun

- $4^{\mathrm{e}}$ étape : collectivement première échange de points de vue littéraires sur l'œuvre à l'oral

- $5^{\mathrm{e}}$ étape : approfondissement de sa lecture personnelle individuellement à l'écrit

- $6^{\mathrm{e}}$ étape : deuxième échange de points de vue littéraires à l'oral

Lors de la cinquième étape, notre objectif est d'approfondir l'approche de l'album-poème en tant qu'iconotexte. Nous avons conçu un questionnaire subjectif progressif, de l'image, au poème, puis à l'iconotexte permettant de mieux comprendre la réception des futurs enseignants. Nous nous sommes appuyée sur le principe défini par G. Langlade et N. Lacelle (2007) lors de leur recherche exploratoire sur la lecture/spectature, selon lequel l'activité d'écriture à partir d'un "questionnaire subjectif» est propice à l'émergence du sujet lecteur/spectateur. Les questions portent tout d'abord sur le rôle des images («Pour vous, quel rôle les images jouent-elles dans ce livre?»); puis sur un élément polysémique du poème, le pronom « elle » ("Qui est "elle" selon vous ? Comment vous la représentez-vous?»); enfin sur la perception de la guerre dans le texte-image («Comment percevez-vous personnellement la guerre dans ce livre?»). Pour chacune de ces questions nous avons recueilli vingt-cinq écrits. Les numéros donnés entre parenthèses ci-dessous correspondent aux numéros des réponses par question. 


\section{Le rôle des images}

L'analyse des réponses à la première question mettra en évidence les représentations du rôle des images dans Les Étoiles sont tombées (David \& Propeck, 2007). Nous envisageons tout d'abord les conceptions des étudiants du rôle des images dans la création de l'œuvre, puis de leur rôle dans la réception de celle-ci.

\section{Que disent les lectrices du rôle des images dans la création de l'œuvre?}

Deux fonctions sont envisagées : l'illustration et l'interprétation. Quatorze étudiantes sur vingt-cinq utilisent «illustration » ou «illustrer » pour définir la fonction des images et 8 autres «complément» ou «compléter». Ces quatorze lectrices conçoivent l'iconographie comme seconde dans la chronologie supposée de la création. Aucune d'entre elles n'imagine que le texte puisse illustrer l'image, ni que les créateurs aient pu réaliser l'œuvre ensemble, dans un projet simultané. On peut penser qu'elles ignorent cette démarche de création ainsi que toute la production d'écrits littéraires sur la peinture ou en relation avec d'autres arts plastiques.

Par ailleurs, le vocable "complète » indique que l'image est perçue comme un ajout. Il nous semble que le terme "complément» est le corollaire de celui d'«illustration". Assujetties aux mots, les images contribueraient à parfaire ou à achever le texte. Différentes expressions expliquent de quelle manière. Premièrement, « représenter ce qui est dit » (réponse 11) signifie une correspondance de l'ordre de la dénotation entre texte et image. Cela est juste pour certains signes verbaux référentiels. Mais on ne saurait parler de redondance, car le mot qui désigne une chose et sa figuration dans l'image ne sont jamais seuls, sinon nous serions dans un imagier. Les autres formulations telles que «permettent une véritable illustration des conséquences de la guerre »(réponse 4), « décrivent la guerre sans trop en dire » (réponse 6), « mettre en scène concrètement les images évoquées dans le texte » (réponse 9), montrent que les mots du poème déforment fortement la perception des photographies. En effet, la guerre n'est jamais présente dans les images, mais la présence concomitante du texte et de l'image conduit les lectrices à voir dans celle-ci ce qui est dit dans le texte, sans que les étudiantes aient conscience de cette déformation.

Deuxièmement, l'image est conçue comme « interprétation » du texte. Quatre étudiantes citent ce mot ou le verbe "interpréter "; deux autres utilisent l'adjectif «symbolique " pour qualifier cette image. On trouve aussi les expressions suivantes: "dimension métaphorique " (réponse 12), «donner du sens " (réponse 16) et "apporter un sens" (réponse 24). Enfin, une lectrice précise que les images «ne sont pas de simples illustrations banales" (réponse 11). La création photographique est perçue comme prenant sa source dans les mots et comme étant une transposition iconographique du texte. Les photographies d'A. Propeck proposeraient une lecture personnelle, le regard subjectif d'une plasticienne et artiste du visuel, sur le poème de F. David.

Comment expliquer l'existence de cette représentation de l'image comme interprétation d'un texte au sein du livre? On peut supposer que les discours contemporains sur l'album qui tendent à valoriser le rôle de l'illustrateur en tant qu'artiste trouvent un écho auprès des futures enseignantes. Cependant ces lectrices ne mentionnent pas la valeur que constitue l'œuvre d'art en soi - le mot "artistique» n'est utilisé qu'une seule fois à 
propos des images (réponse 20) - mais la capacité de l'artiste à signifier autre chose. Une seconde explication pourrait venir de la fréquentation des albums contemporains et notamment de ceux qui sont recommandés par les instances officielles. La complexité de la relation entre texte et image y relève notamment du regard singulier de l'artiste dans le livre.

\section{Que disent les lectrices du rôle des images dans la réception de l'œuvre?}

41 Selon ces vingt-cinq étudiantes, quelles activités les images génèrent-elles? Nous en avons distingué quatre, répertoriées dans le tableau suivant :

Tableau 1. Activités du lecteur

\begin{tabular}{|l|l|}
\hline & Le numéro des écrits correspondant \\
\hline Activité émotionnelle & $3,4,10,11,18,19,21,23$ \\
\hline Activité imageante & $4,7,8$ \\
\hline Activité réflexive & 12 \\
\hline Activité physiologique & 3 \\
\hline
\end{tabular}

Une seule lectrice (réponse 3) mentionne que les images «attirent l'œil » et se révèle sensible à la dimension perceptive des photographies. Elle comprend combien le corps est en jeu dans cette lecture, notamment du côté du visuel, sans échapper pour autant à un stéréotype de l'image : la séduction. Ce faisant, le jeu entre lectrice et image se dit comme un désir. L'une est attirée par l'autre. On trouve plusieurs fois aussi le mot «impact » pour qualifier l'image, comme si le corps du lecteur se trouvait ébranlé au contact de celle-ci.

Mais c'est principalement le cœur, siège symbolique des émotions, qui est touché par les photographies d'A. Propeck. On observe trois effets émotionnels différents :

- soit l'image renforce l'émotion suscitée par le texte, donne du poids, ajoute une intensité (réponses 4, 11, 19);

- soit elle atténue, adoucit cette émotion (réponses 18, 21);

- soit elle fait « vivre des émotions différentes » (réponses 10).

Certaines lectrices précisent les éléments de l'image qui contribuent à ces effets : le noir et blanc ainsi que les objets représentés. Mais la nature des émotions n'est quasiment jamais décrite. Enfin, il est intéressant de souligner des effets émotionnels diamétralement opposés, puisque, pour certaines, les images rendent le texte plus pesant, alors que, pour d'autres, elles créent un soulagement.

Par ailleurs, une seule étudiante mentionne une activité réflexive en disant que les images « induisent un questionnement (sur le choix de leur composition) après la lecture du texte». On voit peut-être décrite ici une stratégie de lecture de l'album qui commencerait par le texte, se poursuivrait par l'observation de l'image et conduirait à une interrogation sur l'agencement des signes iconiques dans la photographie. Cette réflexion semblerait porter sur la démarche de l'artiste vu comme illustrateur. On peut 
s'étonner qu'aucune autre lectrice n'évoque l'image comme source de réflexion, alors que ces photographies nous paraissent extrêmement polysémiques. Peut-être que l'activité réflexive ne dépend pas de l'œuvre elle-même. C'est une attitude vis-à-vis du texte et de l'image à apprendre.

Enfin, les images génèrent chez trois étudiantes d'autres images. Apparaissent alors dans leur discours les mots « imagination » et « imaginer ». Voici les citations :

C'est un peu comme si l'on revenait dans une ville après qu'elle a été ravagée par une tornade. (Écrit 4)

Elles permettent d'imaginer ce que la guerre a pris aux gens, comme la joie d'avoir un vrai gros gâteau d'anniversaire avec sa famille réunie autour. (Écrit 7)

Elles apportent une imagination presque sans limites pour le lecteur qui imagine

des moments précis dans la guerre selon sa propre connaissance. (Écrit 8)

On peut suggérer que la première lectrice fait référence à des images médiatiques. On voit sans doute ici l'influence de l'information visuelle, diffusée par la télévision, qui sert de comparaison pour l'analyse des photographies d'A. Propeck. La deuxième s'arrête sur une double page en particulier et, pour en dire la portée, convoque un souvenir qui appartient à notre culture. Pourtant la photographie de l'artiste ne présente ni gâteau ni famille. Ces motifs sont issus de l'imagination de l'étudiante en relation avec les mots du texte. La dernière étudiante explique, de manière générale, l'activité imageante et indique le lien entre culture personnelle et imagination.

\section{Le pronom « elle » dans le poème}

Dans les réponses à la deuxième question, trois sortes de représentations émergent des écrits : "elle » est "une grand-mère ", «un enfant " ou "une entité » abstraite. Premièrement, douze lectrices identifient le pronom comme évoquant une grand-mère. Les deux acceptions du terme sont présentes. Cette "grand-mère" est définie soit familièrement comme une vieille dame " originale» (réponse 5), « une personne âgée, vieillie par la dureté de la guerre et triste » (réponse 11), « une personne d'un certain âge " (réponse 12), "une très vieille femme " (réponse 17), "une vieille femme" (réponse 21) ; soit, plus fréquemment, comme une aïeule. Elle est alors inscrite dans un système de relations avec d'autres éléments du texte, identifiés à leur tour comme " petit enfant » au singulier (réponses 1, 15 et 19) ou au pluriel (réponses 3, 7, 18 et 20). Des relations familiales entre le locuteur, lui aussi anonyme (ou entre les interlocuteurs de la première double page) et celle dont ce dernier rapporte le discours, sont ainsi créées. Une lectrice imagine même toute une famille potentielle: "Selon moi, "elle" est une grandmère qui a raconté à un de ses petits-enfants comment c'était quand il y avait la guerre en insistant plus sur ce que l'on perdait. Le ou la petit fils ou petite fille en discute ensuite avec un de ses cousins, frères ou sœurs ou amis » (réponse 7).

Ce faisant, ces lectrices semblent construire leur image de ce « elle » en cohérence avec une réalité sociale, la famille, et une valeur, la transmission du passé au sein d'une lignée. Elles activent aussi un stéréotype culturel: la vieille dame qui raconte à des enfants ce qu'elle sait. Enfin, cette grand-mère apparait aussi comme un témoin de la guerre. Une lectrice précise : « une survivante de la guerre [...] (telle la Seconde Guerre mondiale) en étant une enfant à cette époque-là. Peut-être une enfant juive, peut-être une résistante de 14 ans " (réponse 8). Contrairement à d'autres, cette étudiante propose un contexte temporel pour la guerre évoquée dans le texte. C'est pourquoi, sa vision de "elle» s'inscrit dans un univers historique. Elle pense peut-être à Anne Franck ou à Lucie 
Aubrac, et à d'autres bien sûr. En tout cas, tout un réseau référentiel parait ainsi être activé pour permettre aux lectrices de créer une image cohérente autour de ce pronom à l'identité incertaine.

50 La deuxième représentation mentale construite par les lectrices est celle d'un enfant ou d'une petite fille. Trois écrits (réponses 19, 23 et 24) sont concernés, mais un seul donne des détails sur cette figure : "assez rêveuse et en même temps triste " (réponse 24). Il semblerait que cette étudiante projette sa propre émotion sur le personnage qu'elle imagine. Les deux autres écrits précisent les raisons de cette concrétisation imageante. On peut lire : "la façon dont le texte est raconté ressemble aux images d'un enfant » (réponse 19) ; puis, dans un second écrit : « une petite fille qui a connu la guerre et qui la décrit avec ses mots d'enfant » (réponse 23). Dans les deux cas, les lectrices cherchent des indices dans le texte lui-même. Elles s'appuient sur l'énonciation, narration et description, pour identifier qui parle, et voient une petite fille parce qu'elles entendent les mots, les phrases, les énoncés d'une enfant. On peut penser que le rythme, l'anaphore, la brièveté des phrases et l'oralité du texte contribuent à générer cette image. Il nous parait aussi probable que certains objets des photographies (jeu de ficelle, château de cartes, main réalisant une ombre d'animal, ballon, corde, figurines de moutons) présents dans l'imaginaire des lectrices au moment où elles écrivent, participent de cette figuration mentale.

51 Enfin, la troisième représentation n'est pas humaine. Une lectrice identifie « elle » comme « la guerre elle-même » (réponse 2), une autre écrit qu'elle « ne représente pas quelqu'un de vivant ", mais une "entité » abstraite (réponse 6) ; enfin deux autres pensent qu'il s'agit d'une " étoile » (réponses 10 et 25). On peut dire que les étudiantes identifient une allégorie et se réfèrent donc à un savoir littéraire. Sans nommer la figure de rhétorique qui consiste à faire parler une abstraction, elles semblent reconnaitre dans le poème une image linguistique, la prosopopée. Il n'est pas impossible que des représentations lointaines de la guerre en déesse gréco-romaine, Athéna ou Minerve, issues des savoirs scolaires, aient été inconsciemment réactivées. Par ailleurs, l'identification à une étoile trouve une explication par la métaphore. La première lectrice écrit : « Elle voit le monde de là-haut et peut témoigner de tout ce qui s'y passe. Elle raconte la guerre de façon décalée et anecdotique car elle ne la vit pas réellement, elle est seulement témoin » (10); la seconde voit "une étoile représentant une personne qui a vécu la guerre » (réponse 25). Cette conception est issue de l'album-poème lui-même dans lequel le texte et l'image renvoient explicitement aux «étoiles». Cette identification peut être aussi extérieure au texte. Les lectrices feraient référence à des métamorphoses de personnages en étoiles qu'elles auraient pu rencontrer au cours de leurs lectures de récits mythologiques (le mythe de Pégase et de son fils Cérélis) ou de contes comme celui de La Petite Sirène ou de La Petite Fille aux allumettes. Plus largement, l'étoile est le symbole de l'âme humaine dans diverses traditions religieuses. Cette image récurrente expliquerait cette concrétisation des lectrices.

\section{Les représentations de la guerre dans le livre}

La formulation de notre consigne vise à interroger la relation établie entre le lecteur et le texte-image, ce qui explique l'usage du mot «livre». Nous cherchons à savoir quelles réflexions personnelles sur la guerre elle-même une telle œuvre peut susciter chez les sujets lecteurs. Nous proposons donc d'examiner plus particulièrement ici le 
positionnement énonciatif des lectrices. Dans quelle mesure, dans cet écrit, celles-ci parviennent-elles à développer un point de vue personnel ? Nous nous arrêterons ensuite sur les différents aspects de la guerre évoqués. Quelles dimensions affectives et cognitives les étudiantes privilégient-elles dans leur lecture?

Intéressons-nous tout d'abord à la présence du sujet dans ces vingt-cinq réponses pour comprendre si ces lectrices parviennent à adopter un positionnement subjectif. Dans les premiers mots de la réponse, on observe que seulement 6 d'entre elles utilisent les pronoms « je » ou « me »; 5 le pronom impersonnel « on » et 14 ne manifestent aucune implication personnelle dans leur choix énonciatif. Or, si on compare ce positionnement avec celui adopté dans les réponses à la deuxième question sur le pronom « elle », on note une différence de taille. En effet, presque la totalité des réponses dans ce cas-là présente le pronom « je », voire le pronom renforcé « moi ». On peut donc supposer que lorsque le texte est opaque et volontairement polysémique, les étudiantes s'autorisent plus facilement à écrire à la première personne. Sans doute se perçoivent-elles comme réellement responsables de la construction du sens.

En revanche, l'interrogation sur les perceptions de la guerre, malgré l'implication du « vous » et l'usage de l'adverbe "personnellement » dans la consigne, ne permet qu'à un tout petit nombre de prendre la parole en son nom propre. La plupart de celles qui ne s'impliquent pas se retranchent derrière le livre lui-même, c'est-à-dire derrière l'objet lu.

Parmi les six réponses qui adoptent un positionnement énonciatif impliqué personnellement, voyons maintenant quelles dimensions de la guerre leur lecture privilégie. On distingue trois aspects importants. Tout d'abord, une relation entre guerre et émotions douloureuses est formulée (réponses 11 et 19). Une lectrice (réponse 19) explique même ce "sentiment de souffrance et de tristesse » en faisant un lien avec la Seconde Guerre mondiale. Peut-être convoque-t-elle implicitement un vécu personnel en rapport avec sa famille ou des connaissances historiques. On peut penser aussi que c'est le sujet lui-même de la guerre qui suscite la tristesse. Mais cette émotion n'apparait que très peu dans les écrits. Donc lorsqu'il s'agit de développer un point de vue par l'écriture, la tristesse parait mise à distance.

En revanche, et ce sera notre deuxième remarque, la notion de "privation" est mentionnée dans 3 des 6 écrits (réponses 5, 11 et 18). Ces lectrices sont sensibles à l'état de manque représenté dans ce livre. Si on ignore la nature de ce manque dans l'écrit 18, pour la lectrice de l'écrit 6, la perte est autant physique que symbolique : «Dans ce livre, je perçois la guerre comme une privation. Ce qui est évoqué, c'est la perte de l'insouciance, l'enfermement au sens de privation de liberté, la peur, les conditions de vie qui deviennent précaires ».

Quant à la troisième étudiante (réponse 11), elle évoque les conséquences de la privation : «[La guerre] n'apporte que mort et chagrin et enlève le bonheur dans la vie des gens. Rien ne l'arrête et elle ne laisse derrière elle que fantômes et chaos. Elle emprisonne les gens et leur liberté ».

58 Il s'agirait donc d'une lecture à la fois fantasmatique et axiologique, puisque ces écrits mettent en avant l'impression d'insatisfaction que crée la guerre telle qu'elle est évoquée dans le livre, mais aussi les valeurs (bonheur et liberté) dont elle prive ceux qui la subissent.

59 La troisième remarque concerne l'aspect esthétique. Cinq des 6 écrits lient les dimensions précédentes à la forme du livre. En effet, on note une sensibilité aux images poétiques 
(réponse 5) et visuelles (notamment aux signes iconiques dans l'écrit 19), à la description et à la narration (réponses 5 et 6 ), au choix énonciatif (réponses 5 et 18) et au genre (réponse 18). De manière plus générale, une lectrice (réponse 6) évoque le caractère épuré de l'ensemble. Ces étudiantes trouvent donc un intérêt aux propriétés formelles de l'œuvre. Celles-ci ne sont pas mentionnées pour elles-mêmes, mais pour ce qu'elles éveillent chez le lecteur.

Pour conclure sur cette expérience, disons que l'analyse de ces réponses montre le primat du texte sur l'image : bien souvent l'album-poème a été lu comme un album illustré. Le texte est alors considéré comme premier et préexistant à l'image. Au cours de l'expérience, les lectrices commentent les photographies en fonction du texte et jamais l'inverse, ce qui ne les empêche pas d'interpréter l'image. Les échanges qui ont suivi permettent d'apporter des nuances: certaines prennent conscience de l'importance de l'association texte-image, mais rarement de l'interdépendance de l'un et de l'autre. Il faut souligner que cette primauté accordée au poème est sans doute due, en partie, à la discipline dans laquelle s'inscrit le dispositif : la littérature. En effet, le cadre joue un rôle essentiel sur la lecture. On peut penser que ces étudiantes n'ont pas eu beaucoup l'occasion de lire des iconotextes au cours de leurs études secondaires en français, même si l'analyse de l'image est présente depuis quelques décennies dans les programmes de cette discipline.

Poésie, images, école: dans cet article il s'agissait de montrer certains enjeux pour la littérature et l'enseignement, induits par la présence d'un corpus nouveau dans les préconisations depuis une quinzaine d'années. En effet, le livre de poème(s) illustré dont nous avons proposé une typologie, vient enrichir aujourd'hui l'histoire et la création littéraires en général. Il participe également de la reconfiguration des programmes de l'école élémentaire au sein desquels la littérature a trouvé une place à part entière depuis 2002 et l'histoire des arts depuis 2008. Cependant la rencontre avec les œuvres évoquées ne va pas de soi. L'image ajoute un niveau de complexité aux livres de poésie d'autrefois. $\mathrm{Si}$, sans conteste, les livres de poème(s) illustrés suscitent chez les maitres ou chez les futurs professeurs des écoles une vive curiosité ${ }^{6}$ et si la lecture subjective permet un investissement personnel face à ces ouvrages complexes, les difficultés pour lire certains comme des œuvres d'art doubles sont réelles : la réception de la partie visuelle en tant qu'images artistiques reste problématique, la prise en compte de l'interdépendance entre poème et image également. Pourtant nous croyons que l'école est bien le lieu privilégié de ces rencontres multiples entre élèves, poètes et artistes, rencontres avec des êtres, des formes, des paysages, des objets, qui séduisent et ébranlent parfois.

HENRY J.-M. (éd.) (1998). Tour de terre en poésie. Anthologie multilingue de poèmes du monde. Ill. par M. Vautier. Voisins-le-Bretonneux : Rue du monde.

HENRY J.-M. (éd.) (2003). Il pleut des poèmes. Anthologie de poèmes minuscules. Ill. par Zaü.

Voisins-le-Bretonneux : Rue du monde.

HENRY J.-M. (éd.) (2000). Le tireur de langue. Sculptures par R. Roure. Photographieq par Y. Bouvier. Voisins-le-Bretonneux : Rue du monde. 


\section{BIBLIOGRAPHIE}

BALLANGER, F. \& HEISE, S. (1995). « La poésie en morceaux non choisis ». La Revue des livres pour enfants 165 , p. 55-63.

BERTIER, A. (2005). Rêve-moi une lettre. Paris : Éd. MeMo.

BOUTEVIN, C. (2014). Le livre de poème(s) illustré. Étude d'une production littéraire en France de 1995 à nos jours et de sa réception par les professeurs des écoles. Thèse en littérature française : Université Stendhal Grenoble 3.

BOUTEVIN, C. (2015). « Le poème et son illustration dans le recueil contemporain pour la jeunesse ». In : Connan-Pintado, C. \& Béhotéguy, G. (coords), Littérature de jeunesse au présent. Genres littéraires en question(s). Pessac: Presses universitaires de Bordeaux, p. 186-202.

BUtLEN, M. et al. (2008). « De quelques points de résistances dans la mise en place d'un enseignement de la littérature à l'école primaire ». Repères 37, p. 197-226.

BUTOR, M. (2001). Zoo. Ill. par O. Tallec. Voisins-le-Bretonneux : Rue du monde.

CEYSSON, P. (1997). Étude d'une production littéraire. La poésie pour l'enfance et la jeunesse en France de 1970 à 1995. Villeneuve d'Ascq : Presses universitaires du Septentrion.

CHAPON, F. (1987). Le peintre et le livre. L'âge d'or du livre illustré en France, 1870-1970. Paris : Flammarion.

COMBE, D. (2002). « Du "recueil" au "Poème-livre", au "Livre-poème" ». Méthode ! 2, p. 15-22. CONNAN-PINTADO, C. \& POULOU, B. (coords) (2008). L'album contemporain pour la jeunesse. Nouvelles formes, nouveaux lecteurs? Pessac: Presses universitaires de Bordeaux.

DAVID, F. \& GAUTHIER, A. (ill.) (2002). Est-elle Estelle ? Urville-Nacqueville : Møtus. DAVID, F. \& PROPECK, A. (2007). Les Étoiles sont tombées. Urville-Nacqueville : Møtus. DOUMET, C. (2004). Faut-il comprendre la poésie? Paris : Klincksieck. DUbost, J.-P. \& CouPRIE, K. (1998). C'est corbeau. Le Chambon-sur-Ligon : Cheyne. ÉLUARD, P. (1997) [1942]. Liberté. Ill. par C. Goiran. Paris : Père Castor-Flammarion. ÉLUARD, P. (2012) [1942]. Liberté. Ill. par A. Boisrobert et L. Rigaud. Paris : Père Castor-Flammarion. FAVRIAUD, M. et al. (2011). « Le poème du lecteur : mémorisation, imagination, compréhension et image de soi ». In : Mazauric, C., Fourtanier, M.-J. \& Langlade, G. (dirs), Textes de lecteurs en formation. Bruxelles : P. Lang, p. 175-190.

FRAISSE, E. (1997). Les anthologies en France. Paris : Presses universitaires de France.

HUGO, V. (2008a). L'Ogre de Moscovie. Ill. par Pef. Genève : Quiquandquoi.

HUGo, V. (2008b). L'Ogre de Moscovie. Ill. par S. Poliakova. Paris : Gautier-Languereau.

LACELLE, N. \& LANGLADE, G. (2007). « Former des lecteurs/spectateurs par la lecture subjective des œuvres ». In : Dufays, J.-L. (éd.), Enseigner et apprendre la littérature aujourd'hui, pour quoi faire? Sens, utilité, évaluation. Louvain : Presses universitaires de Louvain, p. 55-64. 
LANGLADE, G. \& FOURTANIER, M.-J. (2007). « La question du sujet lecteur en didactique de la lecture littéraire ». In : Falardeau, É. et al. (dirs), La didactique du français. Les voies actuelles de la recherche. Québec : Presses de l'université Laval, p. 101-123.

LANGLET, I. (dir.) (2003). Le recueil littéraire. Pratiques et théorie d'une forme. Rennes, Presses universitaires de Rennes.

LECLAIRE-HALTÉ, A. et al. (2009). « Le rapport texte/image dans le traitement de l'album au cycle 3 : quelques remarques sur les pratiques enseignantes ». In : Canut, E. \& Leclaire-Halté, A. (éds), L'élève et la lecture d'albums. Quelle compréhension du texte et des images? Namur : Presses universitaires de Namur, p. 115-129.

LEFORT, R. (2008). « L'album-poème ». In : Connan-Pintado, C., Gaiotti, F. \& Poulou, B. (coords), L'album contemporain pour la jeunesse. Nouvelles formes, nouveaux lecteurs? Pessac: Presses universitaires de Bordeaux, p. 29-38.

LOUICHON, B. (2008). «L'adaptation : grandeur et misère du patrimoine littéraire ». In : Gondrand, H. \& Vibert, A. (coords), Adapter des œuvres littéraires pour les enfants. Enjeux et pratiques scolaires. Grenoble : SCEREN-CRDP de l'Académie de Grenoble, p. 11-26.

MOEGLIN-DELCROIX, A. (2006). Sur le livre d'artiste. Articles et écrits de circonstance, 1981-2005. Marseille : Le Mot et le reste.

MONTANDON, A. (dir.) (1990). Signe, texte, image. Lyon : Éd. Césura Lyon.

NIÈrES-CHEVReL, I. (2012). « L'album, le mot et la chose ». In : Alary, V. \& Chabrol Gagne, N. (éds), L'album, le parti pris des images. Clermont-Ferrand : Presses universitaires Blaise Pascal, p. 15-20. NORGE (2001). On peut se tromper. Ill. par B. Heitz. Voisins-le-Bretonneux : Rue du monde. PEYRÉ, Y. (2001). Peinture et poésie. Le dialogue par le livre, 1874-2000. Paris : Gallimard. PONGE, F. (1945). Matière et mémoire ou Les lithographies à l'école. Ill. par J. Dubuffet Paris : F. Mourlot. PRÉVERT, J. (2013) [1979]. La Pêche à la baleine. Ill. par H. Galeron. Paris : Gallimard. RANNOU, N. (2010). Le lecteur et son poème. Lire en poésie : expérience littéraire et enjeux pour l'enseignement du français en lycée. Thèse en littératures : université Rennes 2.

RouXeL, A. \& LANGLADE, G. (dir.) (2004). Le sujet lecteur. Lecture subjective et enseignement de la littérature. Actes du colloque, Rennes, les 29, 30 et 31 janvier 2004. Rennes : Presses universitaires de Rennes.

SAINT-JOHN PERSE (1962). L'Ordre des oiseaux. Ill. par G. Braque. Paris : Au vent d'Arles. SERRES, A. (1988). Le Bestiaire des mots. Ill. par M. Mellinette. Chambon-sur-Lignon : Cheyne. SIMÉON, J.-P. (2012). La vitamine P. La poésie, pourquoi, pour qui, comment? Voisins-le-Bretonneux : Rue du monde.

TAUVERON, C. (1999). « Comprendre et interpréter le littéraire à l'école : du texte réticent au texte proliférant ». Repères. Recherches en didactique du français langue maternelle 19, p. 9-38. En ligne : http://www.persee.fr/doc/reper_1157-1330_1999_num_19_1_2289.

VAN DER LINDEN, S. (2006). Lire l'album. Le Puy-en-Velay : L'Atelier du poisson soluble.

VAN DER LINDEN, S. (2013). Album-s. Conflans-Sainte-Honorine/Arles : Éd. de Facto/Actes Sud. VENDEL, E. van de (2014) [2005]. Super Gloupi. Ill. par F. van der Weel. Trad. du néerlandais par C. Bruel. Paris : Éd. Être. 


\section{ANNEXES}

DAVID, F. \& PROPECK, A. (2007). Les Étoiles sont tombées. Urville-Nacqueville : Møtus.

Alors, elle t'a dit?

Elle m'a dit et elle ne m'a pas dit.

Elle t'a dit comment c'est?

Elle m'a dit : «Il n'y a plus la guerre depuis très longtemps ici. Heureusement ! »

Alors, elle ne t'a pas dit !

Elle ne m'a pas dit comment c'est.

Elle m'a dit comment ce n'est plus, quand il y a la guerre.

Quand il y a la guerre, elle m'a dit, plus rien ne tient en équilibre.

Il y a des montagnes de barbelés pour faire tout tenir de force quand il y a la guerre.

Quand il y a la guerre, elle m'a dit, il y a des pièges partout.

Et les ballons légers des jeux sont attrapés comme des rats.

Quand il y a la guerre, elle m'a dit,

on se sent prisonniers, tout le temps,

même de ses vêtements.

Quand il y a la guerre, elle m'a dit,

il n'y a plus de merveilleux nuages.

Ils sont partis. Vers des ciels moins gris.

Quand il y a la guerre, elle m'a dit,

il n'y a plus que de gros nuages lourds.

Quand il y a la guerre, elle m'a dit, il n'y a plus de tendres rires.

Rien que des pleurs et des mouchoirs.

Quand il y a la guerre, elle m'a dit, le temps n'est plus pareil qu'avant.

Il n'y a du temps que pour la guerre tout le temps

quand il y a la guerre.

Quand il y a la guerre, elle m'a dit,

Il n'y a plus de rires joyeux sur la neige.

Et les luges grincent avec des bruits de casseroles.

Quand il y a la guerre, elle m'a dit, il n'y a plus de chiens.

Ou que des chiens devenus fous.

Qui mordent et qui font peur. Ce ne sont plus des chiens si doux.

Ce sont les ombres de ces chiens.

Quand il y a la guerre, elle m'a dit, il n'y a plus d'oranges, plus de jus.

On mange même les pelures quand il y a la guerre.

Quand il y a la guerre, elle m'a dit, les sommeils sont pleins de cauchemars.

On a peur de s'endormir. On a peur des moutons dans la tête.

On ne veut surtout plus les compter quand il y a la guerre. 
Quand il y a la guerre, elle m'a dit, les anniversaires n'ont plus le même goût.

De toute façon, il n'y a plus de gâteaux pour y faire tenir les bougies.

Quand il y a la guerre, elle m'a dit, c'est le bonheur qui est crevé.

Il voudrait tendre un drapeau blanc.

Mais la guerre ne veut rien voir.

Quand il y a la guerre, elle m'a dit,

il n'y a plus de place pour l'amour.

Ni pour les baisers.

Quand il y a la guerre, elle m'a dit,

il ne faut plus rien montrer.

Même pas qu'on est amoureux.

Quand il y a la guerre, elle m'a dit, on porte tout le temps des valises.

Mais on n'emporte que du vent et des feuilles fanées

quand il y a la guerre.

Quand il y a la guerre, elle m'a dit, souvent il n'y a plus de maisons.

Les toits se posent sur les tapis et les tapis se retrouvent sur le sable.

Quand il y a la guerre, elle m'a dit, parfois on saute sur des mines.

Alors on n'a plus de pieds.

Il n'y a plus que les chaussures.

Il n'y a plus que les fantômes qui sont joyeux quand il y a la guerre.

Quand il y a la guerre, elle m'a dit, tout est noir.

Les étoiles sont tombées du ciel.

Elles voudraient remonter, galoper vers leur voie lactée.

Mais elles sont blessées aussi, les étoiles.

C'est seulement quand il n'y a plus la guerre

que la nuit scintille de blancheur.

\section{NOTES}

1. En 1996, le Centre national de documentation pédagogique (CNDP) publie un répertoire d'ouvrages s'adressant aux enfants de deux à onze ans, conçu comme un guide pour les maitres, appelé La Bibliothèque des mille et un livres.

2. Les cycles correspondent à ceux des programmes de 2008.

3. Ministère de l'Éducation nationale, «La poésie à l'école. Littérature » [en ligne], mars 2004 (mis à jour en 2010), [réf. du 22-08-2014]. En ligne: http://media.eduscol.education.fr/file/ ecole/86/1/dossier-poesie_113861.pdf.

4. Ministère de l'Éducation nationale, "Une culture littéraire à l'école. Littérature à l'école. Ressources pour le cycle $3 »$ [en ligne], mars 2008, [réf. du 22-08-2014]. En ligne: http:// media.eduscol.education.fr/file/ecole/46/9/culture-litteraire-ecole_121469.pdf, p. 8.

5. Ministère de l'Éducation nationale (2002), « Horaires et programmes d'enseignement de l'école primaire », Bulletin officiel, numéro hors-série $\mathrm{n}^{\circ} 1$ du 14 février 2002 ; «Littérature au cycle des approfondissements (cycle 3)»(2002), Document d'application des programmes, Paris: Centre national de documentation pédagogique.

6. Nous l'avons montré dans notre thèse (Boutevin, 2014). 


\section{RÉSUMÉS}

Dans un premier temps, cet article a pour objectif de présenter une typologie des livres de poème (s) illustrés, à partir du corpus scolaire préconisé via les listes de référence de l'école élémentaire. Avec les apports de la sémiologie et de la littérature en matière de relation texteimage, quatre types sont distingués : le recueil et l'anthologie illustrés, puis le poème-album et l'album-poème. Dans un second temps, parce que les maitres constituent les premiers lecteurs de ces ouvrages et que la lecture des élèves dépend de la leur, cette contribution interroge la formation des enseignants à la lecture du texte-image poétique et artistique. Pour cela, l'analyse d'un dispositif expérimenté au niveau du master est proposée. Elle met en évidence de quelle manière un échantillon d'étudiants se représente un élément textuel énigmatique extrait d'un album-poème, en prenant plus ou moins en compte l'iconographie et quelles perceptions de la guerre, sujet de l'œuvre retenue, ils ont pu construire grâce à la lecture de cet ouvrage complexe.

First, the aim of this article is to present a typology of the illustrated books of poetry, based on the school corpus advocated through the reference lists of the elementary school. With the contribution of semiology and literature in relation to the text-image relationship, four types are distinguished: the illustrated book and anthology, then the poem-album and the album-poem. Secondly, because the teachers are the first readers of these books and the reading of the pupils depends on their own, this contribution questions the training of teachers in the reading of the poetic and artistic text-image. For this, the analysis of an experimented device at the level of the master is proposed. It shows how a sample of students represents an enigmatic textual element taken from an album-poem, taking more or less account of iconography and what perceptions of war, the subject of the book retained, they were able to build thanks to the reading of this complex album.

\section{INDEX}

Mots-clés : poésie, album, lecture, texte-image, formation des enseignants, sujet lecteur

Keywords : poetry, album, reading, text-image, teacher training, reader topic

\section{AUTEUR}

\section{CHRISTINE BOUTEVIN}

Université de Montpellier, LIRDEF, EA 3749, F-34000, France 